\title{
Type I complex regional pain syndrome (reflex sympathetic dystrophy) developing on the background of carpal tunnel syndrome
}

\begin{abstract}
Reflex sympathetic dystrophy, also known as complex regional pain syndrome is a clinic picture characterized by autonomic failures such as skin edema on limbs, blood flow changes, paleness and sudomotor activity disorders. The syndrome is usually onset after a bone fracture or a tissue and nerve injury due to trauma. 72 year old woman with hypertension, ischemic heart disease, rhythm disorder and mastectomy due to breast cancer, applied to our outpatient clinic through pain and numbness enduring for many years at fingers. At instant exam, bilateral thumb abduction weaknesses were seen and Tinel's sign was positive at right. By the electrophysiological exam, the patient was diagnosed as bilaterally carpal tunnel syndrome distinctly on right. At the control exam the patient reflected that she could not use her hand through swelling and severe pain. At her exam, edema and temperature increase on right hand were determined, pulses were realized and finger movements were limited and painful. Routine blood, electromyography, sympathetic skin responses, cervical imaging, bone scan, serological tests and ultrasound examinations were performed. The findings were well adjusted with reflex sympathetic dystrophy. The patient's examination findings were observed to decline while NSAID and gabapentin treatments were initiated. Carpal tunnel syndrome is an entrapment neuropathy frequently encountered but complex regional pain syndrome development in the presence of this syndrome is rarely seen. In this article, a case with complex regional pain syndrome thought to occur secondary to carpal tunnel syndrome was discussed.
\end{abstract}

Volume 8 Issue 2 - 2018

\author{
Mehmet Güney Senol,' Tansel Kendirli, ${ }^{2}$ \\ Erdem Togrol, ${ }^{2}$ Mehmet Saracoglu ${ }^{3}$ \\ 'Department of Neurology, Hamidiye Șișli Etfal Training Hospital, \\ Turkey \\ ${ }^{2}$ Department of Neurology, Sağlık Bilimleri University, SAUM, \\ Turkey \\ ${ }^{3}$ Department of Neurology, GATA Haydarpașa Training Hospital, \\ Turkey
}

Correspondence: Mehmet Güney SENOL, MD, Șișli Hamidiye Etfal Eğitim ve Araștırma Hastanesi, Nöroloji Kliniği, 3437I, Șișli, Istanbul ,Turkey, Tel +90 $2123735000-3757$, Fax +90 212224 07 72, Email mgsenol@yahoo.com

Received: December 29, 2017 | Published: March 09, 2018

Keywords: reflex sympathetic dystrophy, complex regional pain syndrome, carpal tunnel syndrome

Abbreviations: RSD, reflex sympathetic dystrophy; CRPS, complex regional pain syndrome; CTS, carpal tunnel syndrome; EMG, electromyography

\section{Introduction}

Complex regional pain syndrome (CRPS) is a clinical entity characterized by severe distal extremity pain as well as autonomic signs such as edema and color and skin temperature alterations. It was originally described by Weir Mitchell in 1870 as "causalgia" in order to define symptoms such as severe pain, edema, and color and temperature alterations in the distal extremities of soldiers enduring nervous injury during the American Civil War. Upon the observation of similar clinical manifestations in individuals with no nervous injury, this condition was thought to originate from sympathetic hyperactivity, hence the term "reflex sympathetic dystrophy - RSD" in 1900s. More recently, the term "complex regional pain syndrome" was adopted with consensus in order to clarify the terminological controversy. While "reflex sympathetic dystrophy" defines Type I CRPS, the term "causalgia" is used for defining Type II CRPS.

Carpal tunnel syndrome (CTS) is an entrapment neuropathy frequently encountered but CRPS development in the presence of this syndrome is rarely seen. In this report, a case with CRPS thought to occur secondary to CTS was reviewed with literate.

\section{Case}

A 72-year old female patient attended to our outpatient unit with a long history of pain and numbness in fingers of both hands and right shoulder pain. She had a history of hypertension, ischemic heart disease, rhythm disorder, right mastectomy due to breast cancer 10 years ago, and prosthetic replacement of the right knee joint 2 years ago.

At her initial presentation, she had loss of power in abduction of the $1^{\text {st }}$ finger in both hands with Tinel positivity in the right. A diagnosis of carpal tunnel syndrome (CTS) was made based on these signs and symptoms and the patient was asked to attend monthly follow-up visits. In her initial follow-up visit, she described swelling and severe pain in the right hand as well as inability to use her fingers. Suspension of the arm did not result in an improvement in the pain and swelling. Physical examination showed significant edema of the right hand extending to the forearm, slightly increased skin temperature in the right hand, palpable peripheral pulses, and limited and painful movements in the fingers of the right hand. Despite prescribed therapy with non-steroidal anti-inflammatory agents, anti-depressants, and gabapentin she had no remarkable improvement in her symptoms and sign in her second follow-up visit.

In her third follow-up visit, there was a significant improvement in 
the pain and swelling of the right hand and the patient could move her fingers, reported no numbness, and partial functionality of the right hand in daily chores. Physical examination showed mild edema in the right hand extending to the forearm as well as right thenar atrophy. The $1^{\text {st }}$ finger abduction motor power was $3 / 5$ and $4 / 5$ on the right and left, respectively, with Tinel positivity on the right.

Routine blood tests, EMG, sympathetic skin responses, cervical imaging, bone scintigraphy, serological tests, and ultrasound (breast, venous/arterial, wrist) were performed. Subsequently, the patient was diagnosed as having CRPS based on the results of the diagnostic work-up.

\section{Discussion}

Although CTS is a common entrapment neuropathy, subacute CPRS developing on the background of CTS is a rare clinical occurrence. The syndrome is usually onset after a bone fracture or a tissue and nerve injury due to trauma; besides infections, malignity, ischemic heart and brain diseases and neurologic conditions like multiple sclerosis (MS) may cause the same picture. Other potential etiological factors are shown in Table $1 .^{2}$

Increased local inflammatory response, biochemical mediators and alpha adrenergic receptor activation is charged in pathophysiology. Central and peripheral nervous pathways are both thought to be involved in the formation of the pain, the decisive symptom. Currently, no single hypothesis is thought to account for all clinical signs in the pathophysiology of CRPS (Table 2), and a combined effect on the central and peripheral nervous system is considered to result in the development of clinical manifestations of the condition. ${ }^{3,4}$

Table 1 Causes of complex regional pain syndrome (CRPS)

\begin{tabular}{ll}
\hline Trauma & Malignancy \\
\hline Fractures & Breast \\
Soft tissue injury & Esophagus \\
Iatrogenic & Lung \\
IM/IV injections & Infection \\
Dental extraction & Osteomyelitis \\
Medications & Neurological \\
Ischemic & MS \\
MI & Neuropathy \\
BDH & Arthritic \\
\hline
\end{tabular}

"The International Association for the Study of Pain (IASP)" has defined diagnostic criteria for CRPS-1 (RSD) and CRPS-2 (causalgia). ${ }^{5}$ IASP consensus workshops in 2003, new changes were recommended for clinical diagnostic criteria. (Budapest criteria) (Table 3). ${ }^{6}$

Table 2 Theoretical approaches for CRPS pathophysiology

\author{
Paul Sudeck (1942), increased regional inflammatory responses. \\ Livingstone (1943), increased activity of the cellular pool in medulla spinalis. \\ Nathan (1947) and Drucker (1959), epiphytic transition. \\ Melzack and Wall (1965), gate control theory. \\ Devor (1983), ectopic pacemaker activity of sensory neurons. \\ Roberts (1986),Wide Dynamic Range (WDR) neuronal sensitization. \\ Cooke and Ward (1990), biochemical mediators. \\ Koltzenberg ve McMahon (I99I), alpha adrenergic receptors. \\ Bruehl (1996), psychologic factors \\ Kemler (1999), genetic factors \\ Juottonen (2002), brain plasticity
}

Currently there are no gold standard diagnostic methods for CRPS. Previous studies have suggested that clinical suspicion plays an important role in establishing a diagnosis and electrophysiological studies may be helpful, particularly for the differential diagnosis of CRPS I and II, while autonomic tests (sympathetic skin responses, quantitative sensory responses etc.) may offer limited help; other investigations such as conventional radiography, bone scintigraphy, bone densitometry, and MRI may assist in diagnosing late-stage disease through demonstration of a decreased bone density. Also, it has been underscored that biochemical tests (complete blood count, complement fixation test, sedimentation, anti-nuclear antibodies, and rheumatoid factor) are required to rule out other causes. The role of diagnostic infra-red telethermography currently remains controversial. $^{7}$

Acute management of CRPS is mainly based on steroids and physical therapy. Narcotic or non-narcotic analgesics and antiinflammatory agents should be used when required. Also, intra-nasal calcitonin has been reported to be beneficial in these patients. It should also be borne in mind that agents used for the treatment of neuropathic pain may also be prescribed, and concomitant depression in chronic pain may be alleviated with psychotherapy. Mixed results have been reported for sympathetic blockade. ${ }^{8}$ 
Table 3 Clinical diagnostic criteria for CRPS - Budapest Criteria (2003)

I. Continuing pain, which is disproportionate to any inciting event

2. Must report at least one symptom in three of the four following categories:

- Sensory: reports of hyperesthesia and/or allodynia

- Vasomotor: reports of temperature asymmetry and/or skin color changes and/or skin color asymmetry

- Sudomotor/edema: reports of edema and/or sweating changes and/or sweating asymmetry

- Motor/trophic: reports of decreased range of motion and/or motor dysfunction (weakness, tremor, dystonia) and/or trophic changes (hair, nail, skin)

3. Must display at least one sign at time of evaluation in two or more of the following categories:

- Sensory: evidence of hyperalgesia (to pinprick) and/or allodynia (to light touch and/or deep somatic pressure and/or joint movement)

- Vasomotor: evidence of temperature asymmetry and/or skin color changes and/or asymmetry

- Sudomotor/edema: evidence of edema and/or sweating changes and/or sweating asymmetry

- Motor/trophic: evidence of decreased range of motion and/or motor dysfunction (weakness, tremor, dystonia) and/or trophic changes (hair, nail, skin)

4. There is no other diagnosis that better explains the signs and symptoms

After a diagnosis of CTS was made, she was prescribed antiinflammatory agents, B vitamins, and wrist splinting. However, due to occurrence of other clinical signs and symptoms during her therapy, the treatment was revised as to include anti-inflammatory agents, an anti-depressant, gabapentin, and physiotherapy. This management strategy resulted in a significant improvement in pain and edema, with partial recovery of the finger movements and electrophysiological improvement in CTS. ${ }^{1}$

The etiopathogenesis of CRPS remains controversial. Although CTS represents the most frequent type of entrapment neuropathy, co-occurrence of these two conditions is rare. ${ }^{3}$ We believe that the clinical manifestations of the patient presented herein are unlikely to be completely independent of CTS, although others factors may also have played a role.

\section{Acknowledgements}

None.

\section{Conflict of interest}

The author declares no conflict of interest

\section{References}

1. Stanton-Hicks M. Complex regional pain syndrome. Anesthesiol Clin
North America. 2003;21(4):733-744.

2. Sebastin SJ. Complex regional pain syndrome. Indian J Plast Surg. 2011;44(2):298-307.

3. Goris RJA. Reflex sympathetic dystrophy: model of a severe regional inflamatory response syndrome. World J Surg. 1998;22(2):197-202.

4. Bruehl S. An update on the pathophysiology of complex regional pain syndrome. Anesthesiology. 2010;113(3):713-25.

5. Amadio PC, Mackinnon SE, Merritt WH, et al. Reflex sympathetic dystrophy syndrome: consensus report of an ad hoc committee of the American Association for Hand Surgery on the definition of reflex sympathetic dystrophy syndrome. Plast Reconstr Surg 1991;87(2):371-375.

6. Harden RN, Bruehl S, Perez RSGM. Validation of proposed diagnostic criteria the Budapest Criteria for Complex Regional Pain Syndrome. Pain 2010;150(2):268-274.

7. Turner-Stokes L. Reflex sympathetic dystrophy- a complex regional pain syndrome. Disability and Rehabilitation 2002;9:18:939-947.

8. Goris RJA, Van der Laan L. Reflex Sympathetic Dystrophy - Another View. Eur J Trauma. 2000;27:99-103. 